\title{
Unitary isomorphism of Fock spaces of bosons and fermions arising from a representation of the Cuntz algebra $\mathcal{O}_{2}$
}

\author{
Katsunori Kawamura* \\ College of Science and Engineering Ritsumeikan University, \\ 1-1-1 Noji Higashi, Kusatsu, Shiga 525-8577, Japan
}

\begin{abstract}
Bosons and fermions are described by using canonical generators of Cuntz algebras on any permutative representation. According to branching laws associated with these descriptions, a certain representation of the Cuntz algebra $\mathcal{O}_{2}$ induces Fock representations $\mathcal{H}_{B}$ and $\mathcal{H}_{F}$ of bosons and fermions simultaneously. From this, a unitary operator $U$ from $\mathcal{H}_{B}$ to $\mathcal{H}_{F}$ is obtained. We show the explicit formula of the action of $U$ on the standard basis of $\mathcal{H}_{B}$. It is shown that $U$ preserves the particle number of $\mathcal{H}_{B}$ and $\mathcal{H}_{F}$.
\end{abstract}

Mathematics Subject Classifications (2000). 46K10, 81T05

Key words. recursive boson system, recursive fermion system, Cuntz algebra, branching law.

\section{Introduction}

We have studied relations among representations of Cuntz algebras, bosons and fermions from a standpoint of branching law. Even to this day, algebras of bosons and fermions are most fundamental ingredients in quantum field theory [23, 25]. Their Fock representations are basic and essential in several models. Therefore it is expected that a mathematical study of these representations give a new point of view to quantum field theory. In this paper, we show their relations by using the representation theory of Cuntz

*Electronic mail: kawamura@kurims.kyoto-u.ac.jp. 
algebras. A unitary operator from the Bose-Fock space to the Fermi-Fock space is constructed by using a certain representation of the Cuntz algebra $\mathcal{O}_{2}$ which preserves particle number.

\subsection{Motivation}

We explain our motivation without mathematical definitions in this subsection. Rigorous definitions will be given in $\S 1.2$ and $\S 2$.

\subsubsection{Fermions and bosons described by Cuntz algebras}

In [1] and [19], we have described fermions and bosons by using Cuntz algebras $\mathcal{O}_{2}$ and $\mathcal{O}_{\infty}$, respectively. Fermions are described as polynomials in canonical generators of $\mathcal{O}_{2}$ and their conjugates. Hence the algebra of fermions is embedded into $\mathcal{O}_{2}$ as a unital *-subalgebra. Bosons are described as formal power series in canonical generators of $\mathcal{O}_{\infty}$ and their conjugates. This does not mean that the algebra of bosons is embedded into $\mathcal{O}_{\infty}$. However, these formal sums make sense on a certain dense subspace of any permutative representations of $\mathcal{O}_{\infty}$. These two descriptions enable studies of restrictions of representations of $\mathcal{O}_{2}$ and $\mathcal{O}_{\infty}$ on fermions and bosons. We have shown that their Fock representations are derived from certain representations of these Cuntz algebras.

On the other hand, $\mathcal{O}_{\infty}$ is embedded into $\mathcal{O}_{2}$ by using a certain unital *-embedding such that canonical generators of $\mathcal{O}_{\infty}$ are written as monomials in those of $\mathcal{O}_{2}$. With respect to this embedding, the restriction of any permutative representation of $\mathcal{O}_{2}$ is also a permutative representation of $\mathcal{O}_{\infty}$. This implies that any permutative representation of $\mathcal{O}_{2}$ can be restricted on bosons also. From these, both fermions and bosons are described by using canonical generators of $\mathcal{O}_{2}$. Hence we have an extreme interest in the comparison between fermions and bosons which are simultaneously represented on a given representation space of $\mathcal{O}_{2}$.

\subsubsection{Bosonization, fermionization and boson-fermion correspon- dence}

In physics, a description of a given (algebraic) system by using bosons (resp. fermions) is called bosonization [20, 27] (resp. fermionization [6]). Especially, fermions and bosons are often rewritten each other in some senses. In this case, a pair of bosonization and fermionization is called a boson-fermion correspondence [15, 22, 24] in the broad sense of the term. 


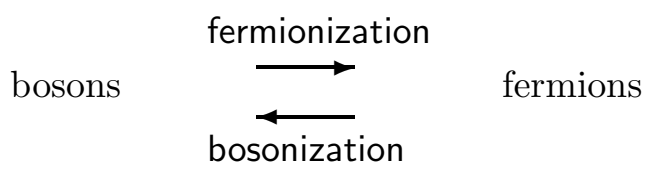

These metamorphoses are not only interesting phenomena but also important techniques in physics [9, 21]. On the other hand, such descriptions are not well-understood as mathematics. In almost all cases, a bosonization (or a fermionization) is done as a computation technique. Therefore nobody has given its mathematical meaning. The algebra of bosons and that of fermions are neither isomorphic nor embedded each other. Hence neither bosonization nor fermionization are executed in a purely algebraic sense without any representation. Remark that infinite sums and normal orders of elements in these algebras make no sense as elements in algebras. These descriptions are usually given on a certain representation space with a certain operator topology or as a formal operation [14, 22, 24]. Hence a mathematical generalization of these is stated as follows:

Problem 1.1 Let $A_{1}$ and $A_{2}$ be algebras such that there is no embedding of one to the other. Let $V$ be a vector space and let $\pi_{i}$ be a representation of $A_{i}$ on $V$ for $i=1,2$.

(i) For $x \in A_{1}$, write $\pi_{1}(x)$ by using elements in $\pi_{2}\left(A_{2}\right)$ (and some exclusive operation) as a rigorous mathematical statement.

(ii) If (i) is done, then characterize such description in some senses.

If $\pi_{2}$ is irreducible, then we can always give an answer to Problem 1.1(i) by using the strong operator topological limit ([19], $\S$ I.A.2) when $V$ is a Hilbert space.

In order to avoid difficulties about operator-valued distributions and physical assumptions, we consider a boson-fermion correspondence between the Bose-Fock space and the Fermi-Fock space as the simplest example in this paper.

\subsection{Recursive boson system and recursive fermion system}

We briefly explain recursive boson system and recursive fermion system in this subsection. For $2 \leq N \leq \infty$, let $\mathcal{O}_{N}$ denote the Cuntz algebra [10]. Let $\left\{s_{n}: n \in \mathbf{N}\right\}$ and $\left\{t_{1}, t_{2}\right\}$ denote the canonical generators of $\mathcal{O}_{\infty}$ and $\mathcal{O}_{2}$, 
respectively, that is, they satisfy that

$$
\begin{array}{ll}
s_{i}^{*} s_{j}=\delta_{i j} I \quad(i, j \in \mathbf{N}), \quad & \sum_{m=1}^{k} s_{m} s_{m}^{*} \leq I \quad(\text { for any } k \geq 1), \\
t_{i}^{*} t_{j}=\delta_{i j} I \quad(i, j=1,2), & t_{1} t_{1}^{*}+t_{2} t_{2}^{*}=I,
\end{array}
$$

where $\mathbf{N} \equiv\{1,2,3, \ldots\}$. Let $\left\{b_{n}: n \in \mathbf{N}\right\}$ and $\left\{a_{n}: n \in \mathbf{N}\right\}$ denote bosons and fermions, that is,

$$
\begin{gathered}
b_{n} b_{m}^{*}-b_{m}^{*} b_{n}=\delta_{n m} I, \quad b_{n} b_{m}-b_{m} b_{n}=b_{n}^{*} b_{m}^{*}-b_{m}^{*} b_{n}^{*}=0, \\
a_{n} a_{m}^{*}+a_{m}^{*} a_{n}=\delta_{n m} I, \quad a_{n} a_{m}+a_{m} a_{n}=a_{n}^{*} a_{m}^{*}+a_{m}^{*} a_{n}^{*}=0
\end{gathered}
$$

for each $n, m \in \mathbf{N}$. We described $\left\{b_{n}: n \in \mathbf{N}\right\}$ and $\left\{a_{n}: n \in \mathbf{N}\right\}$ by using $\left\{s_{n}: n \in \mathbf{N}\right\}$ and $\left\{t_{1}, t_{2}\right\}$ in [19, 1], respectively as follows:

$$
\begin{array}{lll}
b_{1}=\sum_{m=1}^{\infty} \sqrt{m} s_{m} s_{m+1}^{*}, & b_{n}=\rho\left(b_{n-1}\right) & (n \geq 2), \\
a_{1}=t_{1} t_{2}^{*}, & a_{n}=\zeta\left(a_{n-1}\right) & (n \geq 2)
\end{array}
$$

where

$$
\begin{aligned}
& \rho(x)=\sum_{m=1}^{\infty} s_{m} x s_{m}^{*} \quad\left(x \in \mathcal{O}_{\infty}\right), \\
& \zeta(y)=t_{1} y t_{1}^{*}-t_{2} y t_{2}^{*} \quad\left(y \in \mathcal{O}_{2}\right) .
\end{aligned}
$$

We call these descriptions of $\left\{b_{n}: n \in \mathbf{N}\right\}$ and $\left\{a_{n}: n \in \mathbf{N}\right\}$ as the recursive boson system $(=R B S)$ and the recursive fermion system (=RFS), respectively.

Remark 1.2 (i) Remark that $\left\{b_{n}: n \in \mathbf{N}\right\}$ in (1.5) and $\rho(x)$ in (1.7) are not well-defined in $\mathcal{O}_{\infty}$, but they make sense as operators on a certain dense subspace of any permutative representation of $\mathcal{O}_{\infty}$ by Fact 1.1 in [19].

(ii) The $*$-algebra $\mathcal{B}$ of bosons can never be embedded into the $*$-algebra $\mathcal{A}$ of fermions $([19], \S 1.1 .1)$. Especially, $\mathcal{B}$ and $\mathcal{A}$ are not $*$-isomorphic. From this, bosonization and fermionization are usually executed as infinite operations on suitable representation spaces with respect to certain operator topologies. 


\subsection{Fock representations arising from a representation of $\mathcal{O}_{2}$}

In this subsection, we show relations among the Bose-Fock representation, the Fermi-Fock representation and a certain representation of $\mathcal{O}_{2}$. Let $\mathcal{B}$ and $\mathcal{A}$ be as in Remark 1.2(ii) and let $\left\{t_{1}, t_{2}\right\}$ be as in (1.2). Assume that $(\mathcal{H}, \pi)$ is a $*$-representation of $\mathcal{O}_{2}$ with a cyclic vector $\Omega$ satisfying

$$
\pi\left(t_{1}\right) \Omega=\Omega .
$$

This representation exists uniquely up to unitary equivalence [7, 11, 12. We will show an example of this in $\S 4.3$. In [1, 19], we proved the following for $(\mathcal{H}, \pi)$ and $\Omega$ in $(1.9)$ :

(i) Define the dense subspace $\mathcal{D}$ of $\mathcal{H}$ by

$$
\mathcal{D} \equiv \operatorname{Lin}\left\langle\left\{t_{i_{1}} \cdots t_{i_{m}} \Omega: i_{1}, \ldots, i_{m}=1,2, m \geq 1\right\}\right\rangle .
$$

Let $\left\{s_{n}: n \in \mathbf{N}\right\}$ be as in (1.1). Assume that $\mathcal{O}_{\infty}$ is embedded into $\mathcal{O}_{2}$ by

$$
s_{m}=t_{2}^{m-1} t_{1} \quad(m \geq 1)
$$

where we define $t_{2}^{0}=I$. From this, we obtain the restriction $\left(\mathcal{H},\left.\pi\right|_{\mathcal{O}_{\infty}}\right)$ of $(\mathcal{H}, \pi)$ on $\mathcal{O}_{\infty}$. Furthermore, (1.5) and (1.7) are rewritten as follows:

$$
\begin{gathered}
b_{1}=\sum_{m=1}^{\infty} \sqrt{m} t_{2}^{m-1} t_{1} t_{1}^{*}\left(t_{2}^{*}\right)^{m}, \quad b_{n}=\rho\left(b_{n-1}\right) \quad(n \geq 2), \\
\rho(x)=\sum_{m=1}^{\infty} t_{2}^{m-1} t_{1} x t_{1}^{*}\left(t_{2}^{*}\right)^{m-1} .
\end{gathered}
$$

By using (1.11), a unital $*$-representation $\pi_{R B S}$ of $\mathcal{B}$ on $\mathcal{D}$ is defined by using (1.5) with respect to $\left\{\left.\pi\right|_{\mathcal{O}_{\infty}}\left(s_{n}\right): n \in \mathbf{N}\right\}$ and their conjugates on $\mathcal{D}$.

(ii) The unital $*$-representation $\pi_{R F S}$ of $\mathcal{A}$ on $\mathcal{H}$ is defined by using (1.6) with respect to $\left\{\pi\left(t_{1}\right), \pi\left(t_{2}\right)\right\}$ and their conjugates.

(iii) Let $\left(\mathcal{H}_{B}, \pi_{B}\right)$ and $\left(\mathcal{H}_{F}, \pi_{F}\right)$ denote the Fock representations of bosons and fermions with vacua $\Omega_{B}$ and $\Omega_{F}$, respectively [8], that is, which

are cyclic vectors of dense subspaces of $\mathcal{H}_{B}$ and $\mathcal{H}_{F}$, respectively and

$$
\pi_{B}\left(b_{n}\right) \Omega_{B}=0, \quad \pi_{F}\left(a_{n}\right) \Omega_{F}=0 \quad \text { for all } n \in \mathbf{N} .
$$


Then there exist two unitaries $V_{B}: \mathcal{H} \rightarrow \mathcal{H}_{B}$ and $V_{F}: \mathcal{H} \rightarrow \mathcal{H}_{F}$ such that

$$
V_{B} \pi_{R B S}(\cdot) V_{B}^{*}=\pi_{B}, \quad V_{F} \pi_{R F S}(\cdot) V_{F}^{*}=\pi_{F},
$$

that is, two unitary equivalences $\pi_{R B S} \cong \pi_{B}$ and $\pi_{R F S} \cong \pi_{F}$ hold, and $V_{B} \Omega=\Omega_{B}$ and $V_{F} \Omega=\Omega_{F}$ (§3.3 of [1] and Proposition 3.2 of [19]).

From these results, we define the unitary $U$ from $\mathcal{H}_{B}$ to $\mathcal{H}_{F}$ by

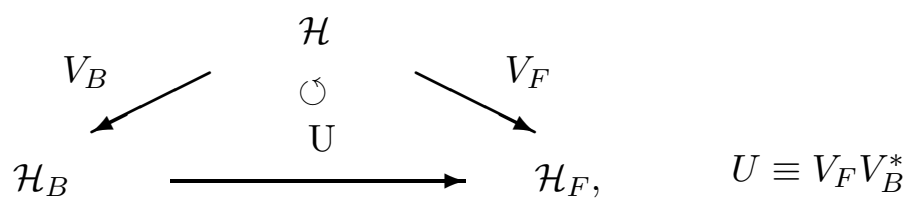

It seems that three triplets $(\mathcal{H}, \pi, \Omega),\left(\mathcal{H}_{B}, \pi_{B}, \Omega_{B}\right)$ and $\left(\mathcal{H}_{F}, \pi_{F}, \Omega_{F}\right)$ are similar in a sense of the representation theory of operator algebras because they are irreducible representations with cyclic vectors $\Omega, \Omega_{B}, \Omega_{F}$ such that they are uniquely determined by algebraic equations with respect to $\Omega, \Omega_{B}, \Omega_{F}$. Remark that both $V_{B}$ and $V_{F}$ in (1.15) are naturally constructed from (1.14). From (1.5) and (1.6), both equations in (1.14) are derived from (1.9). In this sense, (1.9) is most fundamental from the perspective of the representation theory of these algebras.

In spite that two unitaries $V_{B}$ and $V_{F}$ have an established role of unitary equivalence of representations, we can not explain a meaning of the unitary $U$ in (1.16) clearly. By computing $U$ on the standard basis of $\mathcal{H}_{B}$, we consider how similar $\left(\mathcal{H}_{B}, \pi_{B}, \Omega_{B}\right)$ and $\left(\mathcal{H}_{F}, \pi_{F}, \Omega_{F}\right)$ are in the next subsection.

\subsection{Main theorem}

In this subsection, we show our main theorem. Let $\pi_{B}$ and $\pi_{F}$ be as in (1.14). We identify $\pi_{B}\left(b_{n}\right)$ and $\pi_{F}\left(a_{n}\right)$ with $b_{n}$ and $a_{n}$ for $n \geq 1$, respectively.

Theorem 1.3 (i) The unitary $U$ in (1.16) satisfies that $U \Omega_{B}=\Omega_{F}$ and

$$
U\left(\prod_{i=1}^{m}\left(b_{n_{i}}^{*}\right)^{k_{i}}\right) \Omega_{B}=C \cdot A_{n_{1}-1, k_{1}} A_{n_{2}+k_{1}-1, k_{2}} \cdots A_{n_{m}+k_{1}+\cdots+k_{m-1}-1, k_{m}} \Omega_{F}
$$

for $1 \leq n_{1}<\cdots<n_{m}$ and $k_{1}, \ldots, k_{m} \geq 1$ where $C$ denotes the normalization constant which is given by $C \equiv \sqrt{k_{1} ! \cdots k_{m} !}$ and

$$
A_{n, m} \equiv a_{n+1}^{*} \cdots a_{n+m}^{*} \quad(n, m \geq 1) .
$$


(ii) Let $\mathcal{H}_{B, n}$ and $\mathcal{H}_{F, n}$ denote the subspaces of all $n$-particle states of $\mathcal{H}_{B}$ and $\mathcal{H}_{F}$ for $n \geq 0$, respectively. Then

$$
U \mathcal{H}_{B, n}=\mathcal{H}_{F, n} \quad(n \geq 0),
$$

that is, $U$ satisfies the particle number conservation law between bosons and fermions.

For other isomorphism theorems of Fock spaces, see $\S 14.10$ of [15] and Theorem 5.1 in 22]. From Theorem 1.3, the following holds.

Corollary 1.4 Up to vacuum vectors $\Omega_{B}, \Omega_{F}$ and the normalization constant, monomials of bosons and fermions are one-to-one corresponded in the Fock space as follows:

$$
\left(b_{n_{1}}^{*}\right)^{k_{1}} \cdots\left(b_{n_{m}}^{*}\right)^{k_{m}} \mapsto A_{n_{1}-1, k_{1}} \cdots A_{n_{m}+k_{1}+\cdots+k_{m-1}-1, k_{m}} .
$$

Remark 1.5 (i) From Theorem $1.3(\mathrm{i})$, we see that a mode $b_{n}^{*}$ of a boson is nearly corresponded with a block $A_{n^{\prime}, m^{\prime}}$ of fermions in (1.18).

(ii) The statement of Theorem 1.3(i) is a result of the definition of $U$ in (1.16). Conversely, (1.17) defines the unitary operator $U$ from $\mathcal{H}_{B}$ to $\mathcal{H}_{F}$ without the use of representations of Cuntz algebras.

(iii) From Theorem 1.3(ii), number operators $N_{B}$ and $N_{F}$ of bosons and fermions on their Fock spaces are transformed as $U N_{B}=N_{F} U$ [8].

(iv) Theorem 1.3 is an example of branching law (without nontrivial branch) of $\mathcal{O}_{2}, \mathcal{A}$ and $\mathcal{B}$. We will show other branching laws in $\S 3.5$.

Remark 1.6 Since every dimensions of representation spaces $\mathcal{H}_{B}, \mathcal{H}_{F}, \mathcal{H}$ in $\S 1.3$ are countably infinite, it is clear that there exists a one-to-one correspondence among their standard orthonormal basis. Hence there exist many choices of the correspondence and there is no criterion to choose a correspondence in general. On the other hand, the formula (1.17) is derived from two branching laws of representations associated with the RBS and the RFS. It is surprising that pure representation theoretical results give unique correspondence of state vectors with the physical statement about the particle number conservation.

In $\S 2$, we will introduce representations of $\mathcal{O}_{\infty}, \mathcal{O}_{2}, \mathcal{B}$ and $\mathcal{A}$. In $\S 3$, we will explain branching laws and show relations among representations in $\S$ 2. In $\S$ 3.5, we will show the proof of Theorem 1.3, In $\S$, we will show examples. 


\section{Representations of algebras}

In this section, we introduce representations of $\mathcal{O}_{\infty}, \mathcal{O}_{2}, \mathcal{B}$ and $\mathcal{A}$ independently and show their properties.

\subsection{Permutative representations of Cuntz algebras}

For $N=2,3, \ldots,+\infty$, let $\mathcal{O}_{N}$ denote the Cuntz algebra [10], that is, a $\mathrm{C}^{*}$ algebra which is universally generated by $s_{1}, \ldots, s_{N}$ satisfying $s_{i}^{*} s_{j}=\delta_{i j} I$ for $i, j=1, \ldots, N$ and

$$
\sum_{i=1}^{N} s_{i} s_{i}^{*}=I \quad(\text { if } N<+\infty), \quad \sum_{i=1}^{k} s_{i} s_{i}^{*} \leq I, \quad k=1,2, \ldots \quad(\text { if } N=+\infty)
$$

where $I$ denotes the unit of $\mathcal{O}_{N}$.

In $\S 2.2$ of [13], a Cuntz algebra-like object appears. Gopakumar and Gross call it the Cuntz algebra. They regard that this algebra corresponds with (Maxwell-) Boltzmann statistics ([26], p 362). According to their interpretation, we illustrate relations between algebras and statistics as follows:

\begin{tabular}{c|c|c|c}
\hline algebra & boson & fermion & $\mathcal{O}_{N}$ \\
\hline statistics & Bose-Einstein & Fermi-Dirac & Maxwell-Boltzmann(?) \\
\hline
\end{tabular}

Since $\mathcal{O}_{N}$ is simple, that is, there is no nontrivial closed two-sided ideal, any unital homomorphism from $\mathcal{O}_{N}$ to a $\mathrm{C}^{*}$-algebra is injective. If $t_{1}, \ldots, t_{n}$ are elements of a unital $\mathrm{C}^{*}$-algebra $\mathfrak{A}$ such that $t_{1}, \ldots, t_{n}$ satisfy the relations of canonical generators of $\mathcal{O}_{N}$, then the correspondence $s_{i} \mapsto t_{i}$ for $i=1, \ldots, N$ is uniquely extended to a $*$-embedding of $\mathcal{O}_{N}$ into $\mathfrak{A}$ from the uniqueness of $\mathcal{O}_{N}$. Therefore we call such a correspondence among generators by an embedding of $\mathcal{O}_{N}$ into $\mathfrak{A}$.

Define $X_{N} \equiv\{1, \ldots, N\}$ for $2 \leq N<\infty$ and $X_{\infty} \equiv \mathbf{N}$. For $N=$ $2, \ldots, \infty$ and $k=1, \ldots, \infty$, define the product set $X_{N}^{k} \equiv\left(X_{N}\right)^{k}$ of $X_{N}$. Let $\left\{s_{n}: n \in X_{N}\right\}$ denote the set of canonical generators of $\mathcal{O}_{N}$ for $2 \leq N \leq \infty$.

Definition $2.1 \quad$ (i) A representation $(\mathcal{H}, \pi)$ of $\mathcal{O}_{N}$ is permutative if there exists an orthonormal basis $\mathcal{E} \equiv\left\{e_{n}: n \in \Lambda\right\}$ of $\mathcal{H}$ such that $\pi\left(s_{i}\right) \mathcal{E} \subset$ $\mathcal{E}$ for each $i \in X_{N}[7,11$, 12].

(ii) For $J=\left(j_{l}\right)_{l=1}^{k} \in X_{N}^{k}$ with $1 \leq k<\infty$, let $P_{N}(J)$ denote the class of representations $(\mathcal{H}, \pi)$ of $\mathcal{O}_{N}$ with a cyclic unit vector $\Omega \in \mathcal{H}$ such that $\pi\left(s_{J}\right) \Omega=\Omega$ and $\left\{\pi\left(s_{j_{l}} \cdots s_{j_{k}}\right) \Omega\right\}_{l=1}^{k}$ is an orthonormal family in $\mathcal{H}$ where $s_{J} \equiv s_{j_{1}} \cdots s_{j_{k}}$. 
We call the vector $\Omega$ in Definition 2.1 by the $G P$ vector of $(\mathcal{H}, \pi)$.

Results of these classes are shown as follows. For any $J, P_{N}(J)$ is a class of permutative representations, which contains only one unitary equivalence class. From this, we can always identify $P_{N}(J)$ with a representative of $P_{N}(J)$. The class $P_{N}(J)$ is equivalent to $P_{N}(\sigma J)$ where $\sigma J=\left(j_{\sigma(1)}, \ldots, j_{\sigma(k)}\right)$ for any cyclic permutation $\sigma \in \mathbf{Z}_{k}$ for $J=\left(j_{1}, \ldots, j_{k}\right)$. The class $P_{N}(J)$ is irreducible if and only if $\sigma J \neq J$ for any cyclic permutation $\sigma \neq i d$ [7, 11, 12, 17]. Let $P_{N}(j, \ldots, j, k)(p$-times $j$, and $k$ ) denote $P_{N}\left(j^{p} k\right)$ for description of simplicity. We summarize our results as follows.

Lemma 2.2 [7, 17] Let $\mathcal{R} \equiv\left\{P_{2}\left(2^{p-1} 1\right), P_{2}\left(1^{q-1} 2\right): p, q \geq 1, q \neq 2\right\}$.

(i) Any two of classes in $\mathcal{R}$ are not unitarily equivalent.

(ii) All of classes in $\mathcal{R}$ are irreducible.

Lemma 2.3 ([19], Lemma 2.2) Let $\mathcal{T} \equiv\left\{P_{\infty}(p), P_{\infty}\left(1^{p} 2\right): p \geq 1\right\}$.

(i) Any two of classes in $\mathcal{T}$ are not unitarily equivalent.

(ii) All of classes in $\mathcal{T}$ are irreducible.

\subsection{Representations of bosons}

We summarize several representations of bosons and their properties. Let $\mathcal{B}$ denote the $*$-algebra generated by $\left\{b_{n}: n \in \mathbf{N}\right\}$ which satisfies (1.3). The algebra $\mathcal{B}$ is called the Heisenberg algebra [15, 22], the universal enveloping algebra of the Heisenberg Lie algebra [15], or the Weyl algebra [16]. A representation of $\mathcal{B}$ is a pair $(\mathcal{H}, \pi)$ such that $\mathcal{H}$ is a complex Hilbert space with a dense subspace $\mathcal{D}$ and $\pi$ is a $*$-homomorphism from $\mathcal{B}$ to the $*$-algebra $\left\{x \in \operatorname{End}_{\mathbf{C}}(\mathcal{D}): x^{*} \mathcal{D} \subset \mathcal{D}\right\}$. A cyclic vector of $(\mathcal{H}, \pi)$ is a vector $\Omega \in \mathcal{D}$ such that $\pi(\mathcal{B}) \Omega=\mathcal{D}$.

Definition 2.4 For $\lambda, q \geq 1$ and $i=1, \ldots, q$, let $B F_{q, i}(\lambda)$ denote the class of representations $(\mathcal{H}, \pi)$ of $\mathcal{B}$ with a cyclic vector $\Omega$ satisfying

$$
\pi\left(b_{q(n-1)+i} b_{q(n-1)+i}^{*}\right) \Omega=\lambda \Omega, \quad \pi\left(b_{q(n-1)+j}\right) \Omega=0
$$

for $n \in \mathbf{N}$ and $j=1, \ldots, q, j \neq i$.

The classes $B F_{1,1}(p), B F_{2,1}(2)$ and $B F_{2,2}(2)$ are same as $F_{p}, F_{21}$ and $F_{12}$ in Definition 2.2 of [19]. A representation $(\mathcal{H}, \pi)$ of $\mathcal{B}$ is called irreducible if there exists a $\pi(\mathcal{B})$-invariant dense subspace $\mathcal{D}$ of $\mathcal{H}$ such that if a linear operator $y$ from $\mathcal{D}$ to $\mathcal{D}$ satisfying $y \pi(x)=\pi(x) y$ on $\mathcal{D}$ for any $x \in \mathcal{B}$, is a scalar multiples of $I$. 
Lemma 2.5 ([19], Lemma 2.2) Let $\mathcal{S} \equiv\left\{B F_{1,1}(p), B F_{1,2}(2), B F_{2,1}(2): p \geq\right.$ $1\}$.

(i) For each $S \in \mathcal{S}$, any two representations belonging to $S$ are unitarily equivalent. From this, we can identify a representation belonging to $S \in \mathcal{S}$ with $S$.

(ii) Any two of classes in $\mathcal{S}$ are not unitarily equivalent.

(iii) All of classes in $\mathcal{S}$ are irreducible.

We see that $B F_{1,1}(1)$ is the Fock representation of $\mathcal{B}$ with the vacuum $\Omega$.

\subsection{Representations of fermions}

Let $\mathcal{A}$ denote the $*$-algebra generated by $\left\{a_{n}: n \in \mathbf{N}\right\}$ which satisfies (1.4). The algebra $\mathcal{A}$ is isomorphic to the Clifford algebra [15, 22]. The $\mathrm{C}^{*}-$ algebra $\mathrm{A}$ universally generated by $\mathcal{A}$ is called the $C A R$ algebra [8]. Every *-representation $\mathcal{A}$ is uniquely extended to the unital *-representation of $\mathrm{A}$. The $\mathrm{C}^{*}$-algebra $\mathrm{A}$ is simple.

Definition 2.6 (i) For $p \geq 1$ and $1 \leq i \leq p$, let $F F_{p, i}$ denote the class of representations $(\mathcal{H}, \pi)$ of $\mathcal{A}$ with a cyclic vector $\Omega$ satisfying

$$
\pi\left(a_{p(n-1)+i}\right) \Omega=\pi\left(a_{p(n-1)+j}^{*}\right) \Omega=0
$$

for $n \geq 1, j=1, \ldots, p, j \neq i$.

(ii) For $p \geq 1$ and $1 \leq i \leq p$, let $F F_{p, i}^{*}$ denote the class of representations $(\mathcal{H}, \pi)$ of $\mathcal{A}$ with a cyclic vector $\Omega$ satisfying

$$
\pi\left(a_{p(n-1)+i}^{*}\right) \Omega=\pi\left(a_{p(n-1)+j}\right) \Omega=0
$$

for $n \geq 1, j=1, \ldots, p, j \neq i$.

By definition, $F F_{2,1}=F F_{2,2}^{*}$ and $F F_{2,2}=F F_{2,1}^{*}$.

Example 2.7 (i) When $p=i=1$,

$$
\pi\left(a_{n}\right) \Omega=0 \quad(n \geq 1) .
$$

Hence $F F_{1,1}$ is the Fock representation of $\mathcal{A}$ with the vacuum $\Omega$. Every representation $F F_{p, i}$ and $F F_{p, i}^{*}$ are obtained from the Bogoliubov transformation of the $F F_{1,1}\left[\underline{8}\right.$. Hence $F F_{p, i}$ and $F F_{p, i}^{*}$ are often called the Fock representation in the broad sense of the term even if $(p, i) \neq(1,1)\left[22\right.$. In this paper, we call only $F F_{1,1}$ the Fermi-Fock representation of $\mathcal{A}$. 
(ii) When $p=2, F F_{2,1}$ satisfies

$$
\pi\left(a_{2 n-1}\right) \Omega=\pi\left(a_{2 n}^{*}\right) \Omega=0 \quad(n \geq 1) .
$$

Hence $F F_{2,1}$ is the infinite wedge representation of $\mathcal{A}$ by Proposition 3.6 in [18. In the same way, $F F_{2,2}$ is the dual infinite wedge representation of $\mathcal{A}$.

Lemma 2.8 Let $\mathcal{T} \equiv\left\{F F_{p, i}: p \geq 1,1 \leq i \leq p\right\}$.

(i) For each $T \in \mathcal{T}$, any two representations belonging to $T$ are unitarily equivalent. From this, we can identify a representation belonging to $T \in \mathcal{T}$ with $T$.

(ii) Any two of classes in $\mathcal{T}$ are not unitarily equivalent.

(iii) Any class in $\mathcal{T}$ is irreducible.

Proof. (i) From Definition 2.2 and Theorem 2.3 of [5], the statement holds. (ii) We see that $\mathcal{A}$ is a dense $*$-subalgebra of the fixed-point subalgebra $\mathcal{O}_{2}^{U(1)}$ with respect to the gauge action of $\mathcal{O}_{2}$. From Theorem 2.3 in [5] and Proposition 3.6. we see that $F F_{p, i}$ is $P\left[\sigma\left(2^{p-1} 1\right)\right]$ for a certain $\sigma \in \mathbf{Z}_{p}$. Therefore the statement holds.

(iii) Since any class in $\mathcal{T}$ is given as a Bogoliubov transformation from the Fock representation, and the Fock representation is irreducible, the statement holds.

\section{Branching laws}

In this section, we show branching laws among representations of $\mathcal{O}_{\infty}, \mathcal{O}_{2}$, $\mathcal{B}$ and $\mathcal{A}$ in $\S 2$.

\subsection{Introduction to branching laws}

First, we explain the notion of branching law. For a group $G$, if there exists an embedding of $G$ into some other group $G^{\prime}$, then any representation $\pi$ of $G^{\prime}$ induces the restriction $\left.\pi\right|_{G}$ of $\pi$ on $G$. The representation $\left.\pi\right|_{G}$ is not irreducible in general even if $\pi$ is irreducible. If $\left.\pi\right|_{G}$ is decomposed into the direct sum of a family $\left\{\pi_{\lambda}: \lambda \in \Lambda\right\}$ of irreducible representations of $G$, then the equation

$$
\left.\pi\right|_{G}=\bigoplus_{\lambda \in \Lambda} \pi_{\lambda}
$$


is called the branching law of $\pi$. Especially, if $\left.\pi\right|_{G}$ itself is irreducible, then the branching law of $\left.\pi\right|_{G}$ has no nontrivial branch. The branching law can be also considered for a pair $(A, B)$ of a subalgebra $A$ and an algebra $B$. We can consider branching laws for the following pairs:

(i) $\left(\mathcal{O}_{\infty}, \mathcal{O}_{2}\right)$,

(ii) $\left(\mathcal{A}, \mathcal{O}_{2}\right)$,

(iii) $\left(\mathcal{B}, \mathcal{O}_{\infty}\right)$,

(iv) $\left(\mathcal{B}, \mathcal{O}_{2}\right)$

where $\mathcal{B}$ is neither a subalgebra of $\mathcal{O}_{\infty}$ nor that of $\mathcal{O}_{2}$, but branching laws of permutative representations can be considered as if $\mathcal{B}$ was a subalgebra of $\mathcal{O}_{\infty}$ by [19], and we write this inclusion like relation as the symbol " $\rightsquigarrow$ " in the left part of the following diagram. From $\left(\mathcal{O}_{\infty}, \mathcal{O}_{2}\right)$ and $\left(\mathcal{B}, \mathcal{O}_{\infty}\right)$, we can consider branching laws for the pair $\left(\mathcal{B}, \mathcal{O}_{2}\right)$. We illustrate relations among them as follows:

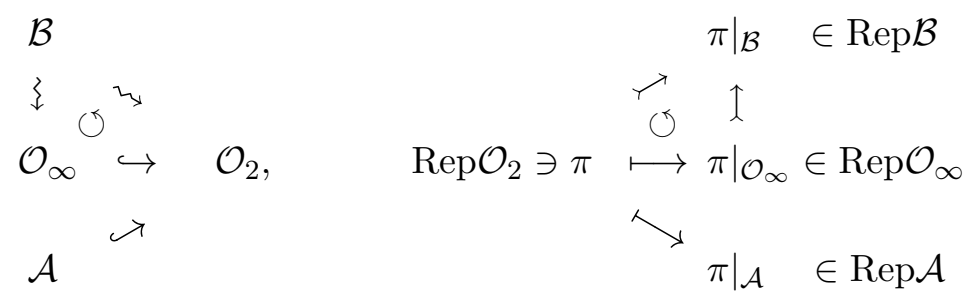

In consequence, we can compare two branching laws for a permutative representation of $\mathcal{O}_{2}$ associated with $\left(\mathcal{B}, \mathcal{O}_{2}\right)$ and $\left(\mathcal{A}, \mathcal{O}_{2}\right)$.

\section{$3.2 \mathcal{O}_{\infty}$ and $\mathcal{O}_{2}$}

Let $\left\{s_{n}: n \in \mathbf{N}\right\}$ and $\left\{t_{1}, t_{2}\right\}$ be as in (1.1) and (1.2). Assume that $\mathcal{O}_{\infty}$ is embedded into $\mathcal{O}_{2}$ by (1.11). From this, $t_{2} s_{n}=s_{n+1}$ for each $n \in \mathbf{N}$.

Proposition 3.1 For $P_{N}(J)$ in Definition 2.1 $($ ii $)$, let $\left.P_{2}(J)\right|_{\mathcal{O}_{\infty}}$ denote the restriction of the (class of) representation $P_{2}(J)$ on $\mathcal{O}_{\infty}$.

(i) For $p \geq 1$,

$$
\left.P_{2}\left(12^{p-1}\right)\right|_{\mathcal{O}_{\infty}}=P_{\infty}(p)
$$

where we define $12^{0}=1$ for convenience.

(ii) For $q \geq 1$,

$$
\left.P_{2}\left(1^{q} 2\right)\right|_{\mathcal{O}_{\infty}}=P_{\infty}\left(1^{q-1} 2\right)
$$

where we define $1^{0} 2=2$ for convenience. 
Proof. (i) Fix $p \geq 1$. Let $(\mathcal{H}, \pi)$ be $P_{2}\left(12^{p-1}\right)$ with the GP vector $\Omega$. We identify $\pi\left(t_{i}\right)$ and $t_{i}$ for $i=1,2$. Then $t_{1} t_{2}^{p-1} \Omega=\Omega$. Define $\Omega^{\prime} \equiv t_{2}^{p-1} \Omega$. Then $\Omega^{\prime}$ is a cyclic vector and $s_{p} \Omega^{\prime}=\Omega^{\prime}$. Let $V \equiv \operatorname{Lin}\left\langle\left\{t_{J} \Omega^{\prime}: J \in\{1,2\}^{*}\right\}\right\rangle$ where $\{1,2\}^{*} \equiv \bigcup_{l \geq 1}\{1,2\}^{l}$. Then we see that $V$ is a dense subspace of $\mathcal{H}$.

Fix $p \geq 1$. For $n_{2}, \ldots, n_{k}, m_{1}, \ldots, m_{k-1} \geq 1$ and $n_{1}, m_{k} \geq 0$, let $J=\left(1^{n_{1}} 2^{m_{1}} \cdots 1^{n_{k}} 2^{m_{k}}\right) \in\{1,2\}^{*}$. Then

$$
t_{J} \Omega^{\prime}=s_{1}^{n_{1}} \cdot s_{m_{1}+1} s_{1}^{n_{2}-1} \cdot s_{m_{2}+1} s_{1}^{n_{3}-1} \cdots s_{m_{k-1}+1} s_{1}^{n_{k}-1} s_{m_{k}+p} \Omega^{\prime} .
$$

From this, $t_{J} \Omega^{\prime} \in \mathcal{O}_{\infty} \Omega^{\prime}$ for each $J \in\{1,2\}^{*}$. Therefore $V \subset \mathcal{O}_{\infty} \Omega^{\prime}$. This implies $\mathcal{H}=\overline{\mathcal{O}_{\infty} \Omega^{\prime}}$. Hence $\left(\mathcal{H},\left.\pi\right|_{\mathcal{O}_{\infty}}\right)$ is $P_{\infty}(p)$.

(ii) Assume that a vector $\Omega$ satisfies $t_{1^{q} 2} \Omega^{\prime}=\Omega^{\prime}$. Define $\Omega \equiv t_{1^{q-1}{ }_{2}} \Omega^{\prime}$. Then $s_{1}^{q-1} s_{2} \Omega=t_{1}^{q-1} t_{2} t_{1} \Omega=\Omega$. Hence $V \equiv \overline{\mathcal{O}_{\infty} \Omega}$ is $P_{\infty}\left(1^{q-1} 2\right)$. On the other hand, $t_{1} \Omega=s_{1} \Omega$ and $t_{2} \Omega=t_{2} t_{1^{q-1}{ }_{2}} t_{1} \Omega=s_{2} s_{1^{q-2}} s_{2} \Omega$. From these, $t_{i} \Omega \in V$ for $i=1,2$. Furthermore, we see that

$$
t_{1} s_{j_{1}} \cdots s_{j_{k}} \Omega=s_{1} s_{j_{1}} \cdots s_{j_{k}} \Omega, \quad t_{2} s_{j_{1}} \cdots s_{j_{k}} \Omega=s_{j_{1}+1} s_{j_{2}} \cdots s_{j_{k}} \Omega .
$$

Therefore $t_{J} \Omega \in V$ for each $J \in\{1,2\}^{*}$. Since $\Omega$ is a cyclic vector of $P_{2}\left(1^{q} 2\right)$, the statement holds.

\subsection{Bosons and $\mathcal{O}_{2}$}

We show relations among representations of boson and $\mathcal{O}_{2}$ according to (1.5) and (1.11). From (1.5),

$$
s_{n} b_{m}=b_{m+1} s_{n}, \quad s_{n} b_{m}^{*}=b_{m+1}^{*} s_{n} \quad(n, m \in \mathbf{N}) .
$$

Proposition $3.2 \quad$ (i) For $p \geq 1,\left.P_{2}\left(12^{p-1}\right)\right|_{\mathcal{B}}=B F_{1,1}(p)$.

(ii) For $p \geq 1,\left.P_{\infty}\left(1^{p-1} 2\right)\right|_{\mathcal{B}}=B F_{p, 1}(2) \oplus \cdots \oplus B F_{p, p}(2)$.

(iii) For $p \geq 1,\left.P_{2}\left(1^{p} 2\right)\right|_{\mathcal{B}}=B F_{p, 1}(2) \oplus \cdots \oplus B F_{p, p}(2)$.

Proof. (i) From Proposition [3.1(i) and $\left.P_{\infty}(p)\right|_{\mathcal{B}}=B F_{1,1}(p)$ by Theorem 1.1 of [19], the statement holds.

(ii) When $p=1$, the statement is proved by Theorem 1.1 of [19]. Assume $p \geq 2$. Let $\Omega$ be a non-zero vector satisfying $s_{1}^{p-1} s_{2} \Omega=\Omega$. Define

$$
\Omega_{1} \equiv \Omega, \quad \Omega_{2} \equiv s_{1}^{p-2} s_{2} \Omega, \ldots, \quad \Omega_{p-1} \equiv s_{1} s_{2} \Omega, \quad \Omega_{p} \equiv s_{2} \Omega
$$


and $T_{i} \equiv s_{1}^{p-i} s_{2} s_{1}^{i-1}$ where $s_{1}^{0} \equiv I$. Then $T_{i} \Omega_{i}=\Omega_{i}$ for each $i=1, \ldots, p$. We see that $b_{p-j+1} \Omega_{i}=\delta_{i, j} s_{1}^{p} \Omega_{i}$ for $i, j=1, \ldots, p$. From this,

$$
b_{p(n-1)+p-j+1} \Omega_{i}=\delta_{i j} T_{i}^{n-1} s_{1}^{p} \Omega_{i} \quad(i, j=1, \ldots, p, n \geq 1) .
$$

Moreover $b_{p(n-1)+p-i+1}^{*} b_{p(n-1)+p-i+1} \Omega_{i}=\Omega_{i}$ for $i=1, \ldots, p, n \geq 1$. Hence $V_{i} \equiv \mathcal{B} \Omega_{i}$ is $B F_{p, p-i+1}(2)$ for each $i=1, \ldots, p$. Therefore $W \equiv V_{1} \oplus \cdots \oplus V_{p}$ is $B F_{p, 1}(2) \oplus \cdots \oplus B F_{p, p}(2)$.

On the other hand,

$$
\begin{gathered}
s_{1} \Omega_{1}=b_{1} \Omega_{p}, \quad s_{1} \Omega_{i}=\Omega_{i-1}, \quad s_{2} \Omega_{1}=\Omega_{p}, \quad s_{2} \Omega_{i}=b_{1}^{*} \Omega_{i-1}, \\
s_{n} \Omega_{1}=\{(n-2) !\}^{-1 / 2}\left(b_{1}^{*}\right)^{n-2} \Omega_{p}, \quad s_{n} \Omega_{i}=\{(n-1) !\}^{-1 / 2}\left(b_{1}^{*}\right)^{n-1} \Omega_{i-1}
\end{gathered}
$$

for $n \geq 3$ and $i=2, \ldots, p$. These imply that $s_{n} \Omega_{i} \in W$ for each $i=1, \ldots, p$ and $n \in \mathbf{N}$. From this and (3.6), $s_{n}\left(a_{n_{1}}^{*}\right)^{k_{1}} \cdots\left(a_{n_{r}}^{*}\right)^{k_{r}} a_{m_{1}}^{l_{1}} \cdots a_{m_{t}}^{l_{t}} \Omega_{i} \in W$ for each $i=1, \ldots, p, n_{1}, \ldots, n_{r}, m_{1}, \ldots, m_{t}, k_{1}, \ldots, k_{r}$ and $l_{1}, \ldots, l_{t}$. Hence $s_{n} W \subset W$ for each $n \in \mathbf{N}$. From this, $s_{J} \Omega=s_{J} \Omega_{1} \in W$ for each $J$. Therefore $W$ is dense in $\mathcal{O}_{\infty} \Omega$. This implies the statement.

(iii) From (ii) and Proposition 3.1(ii), the statement holds.

\subsection{Fermions and $\mathcal{O}_{2}$}

We show relations among representations of fermions and $\mathcal{O}_{2}$. From (1.6),

$$
t_{i} a_{m}=(-1)^{i-1} a_{m+1} t_{i}, \quad(-1)^{i-1} t_{i} a_{m}^{*}=a_{m+1}^{*} t_{i}
$$

for $i=1,2$ and $m \in \mathbf{N}$. For $p \geq 1$, let $(\mathcal{H}, \pi)$ be $P_{2}\left(2^{p-1} 1\right)$ with the GP vector $\Omega$. Define

$$
\Omega_{1} \equiv \Omega, \quad \Omega_{2} \equiv t_{2}^{p-2} t_{1} \Omega, \ldots, \quad \Omega_{p-1} \equiv t_{2} t_{1} \Omega, \quad \Omega_{p} \equiv t_{1} \Omega .
$$

From (3.11), the following is verified

Lemma 3.3 (i) When $p=1, a_{n}^{*} \Omega_{1}=t_{1}^{n-1} t_{2} \Omega_{1}$ for each $n \geq 1$.

(ii) When $p \geq 2, a_{p-i+1}^{*} \Omega_{j}=\delta_{i j}(-1)^{p-i} t_{2}^{p-i+1} \Omega$ for $i, j=1, \ldots, p$.

(iii) When $p \geq 2$, for $i, j=1, \ldots, p, l \geq 2$,

$$
a_{p(l-1)+p-i+1}^{*} \Omega_{j}=\delta_{i j}(-1)^{p-i+(p-1)(l-1)} t_{2}^{p-i} t_{1}\left(t_{2}^{p-1} t_{1}\right)^{l-2} t_{2}^{p} \Omega .
$$

In particular, when $i=j=1$,

$$
a_{p l}^{*} \Omega=(-1)^{(p-1) l}\left(t_{2}^{p-1} t_{1}\right)^{l-1} t_{2}^{p} \Omega, \quad a_{p(l-1)+i}^{*} \Omega=0
$$

for $l \geq 1,1 \leq i \leq p-1$. 
From Lemma 3.3 and (1.4), the following holds.

Lemma 3.4 (i) For $i, j=1, \ldots, p, i \neq j, l \geq 1$,

$$
\begin{aligned}
& a_{p(l-1)+p-i+1} a_{p(l-1)+p-i+1}^{*} \Omega_{i}=\Omega_{i}, \\
& a_{p(l-1)+p-i+1}^{*} a_{p(l-1)+p-i+1} \Omega_{j}=\Omega_{j} .
\end{aligned}
$$

In particular, for $l \geq 1$ and $1 \leq i \leq p-1$,

$$
a_{p l} a_{p l}^{*} \Omega=\Omega, \quad a_{p(l-1)+i}^{*} a_{p(l-1)+i} \Omega=\Omega .
$$

(ii) For $i, j=1, \ldots, p, i \neq j, l \geq 1$,

$$
a_{p(l-1)+p-i+1} \Omega_{i}=a_{p(l-1)+p-i+1}^{*} \Omega_{j}=0 .
$$

Lemma 3.5 For $\left\{\Omega_{j}\right\}_{j=1}^{p}$ in (3.12), the following holds:

$$
t_{1} \Omega_{1}=\Omega_{p}, \quad t_{2} \Omega_{1}=a_{1}^{*} \Omega_{p}, \quad t_{1} \Omega_{j}=a_{1} \Omega_{j-1}, \quad t_{2} \Omega_{j}=\Omega_{j-1} \quad(2 \leq j \leq p) .
$$

Proof. By definition, $t_{1} \Omega_{1}=\Omega_{p}$ and $t_{2} \Omega_{j}=\Omega_{j-1}$ for $2 \leq j \leq p$. On the other hand, $t_{2} \Omega_{1}=t_{2} t_{1}^{*} \Omega_{p}=a_{1}^{*} \Omega_{p}$ and $t_{1} \Omega_{j}=t_{1} t_{2}^{*} \Omega_{j-1}=a_{1} \Omega_{j-1}$ for $2 \leq j \leq p$.

Proposition 3.6 For any $p \geq 1$, the following holds:

$$
\left.P_{2}\left(2^{p-1} 1\right)\right|_{\mathcal{A}}=F F_{p, 1} \oplus \cdots \oplus F F_{p, p},\left.\quad P_{2}\left(1^{p-1} 2\right)\right|_{\mathcal{A}}=F F_{p, 1}^{*} \oplus \cdots \oplus F F_{p, p}^{*} .
$$

Proof. $\quad$ Let $\mathcal{H}$ be the representation space of $P_{2}\left(2^{p-1} 1\right)$. By Lemma 3.3, we see that $\overline{\mathcal{A} \Omega_{j}}$ is $F F_{p, j}$ for each $j=1, \ldots, p$. Therefore $F F_{p, 1} \oplus \cdots \oplus F F_{p, p}$ is a subrepresentation of $\mathcal{H}$. It is sufficient to show that $W \equiv \mathcal{A} \Omega_{1} \oplus \cdots \oplus \mathcal{A} \Omega_{p}$ is dense in $\mathcal{H}$.

By Lemma 3.5, $t_{i} \Omega_{j} \in W$ for each $i=1,2$ and $j=1, \ldots, p$. From this and (3.11),

$$
t_{i} a_{S}^{*} a_{T} \Omega_{j}=(-1)^{(|S|+|T|)(i-1)} a_{S+1}^{*} a_{T+1} t_{i} \Omega_{j} \in W
$$

where $a_{S}^{*}=a_{n_{1}}^{*} \cdots a_{n_{k}}^{*}, a_{T} \equiv\left(a_{T}^{*}\right)^{*}$ and $|S|=k, S+1 \equiv\left\{n_{1}+1, \ldots, n_{k}+1\right\}$ for $S=\left\{n_{1}, \ldots, n_{k}\right\}$. Let $\mathcal{R} \equiv\left\{t_{J} \Omega: J \in\{1,2\}^{*}\right\}$. From Lemma 3.5. $\mathcal{R} \subset W$. Since the linear hull of $\mathcal{R}$ is dense in $\mathcal{H}, W$ is also dense in $\mathcal{H}$. Hence the first statement holds. 
Define the automorphism $\alpha$ of $\mathcal{O}_{2}$ by $\alpha\left(t_{1}\right) \equiv t_{2}$ and $\alpha\left(t_{2}\right) \equiv t_{1}$. Then $P_{2}\left(2^{p-1} 1\right) \circ \alpha=P_{2}\left(1^{p-1} 2\right)$. Furthermore $\left.\alpha\right|_{\mathcal{A}}$ is also an automorphism of $\mathcal{A}$. We see that $F F_{p, i} \circ \alpha=F F_{p, i}^{*}$. From this and the first statement, the second statement holds.

For other results of representations of fermions and Cuntz algebras, see [1, 2, 3, 4, 5, 18].

\subsection{Bosons and fermions}

In this subsection, we summarize relations among representations of algebras $\mathcal{O}_{2}, \mathcal{B}$ and $\mathcal{A}$, and prove Theorem 1.3. From Proposition 3.2 and 3.6, we obtain the unitary $U_{p}$ from $B F_{1,1}(p)$ to $F F_{p, 1} \oplus \cdots \oplus F F_{p, p}$ for $p \geq 1$ as follows:

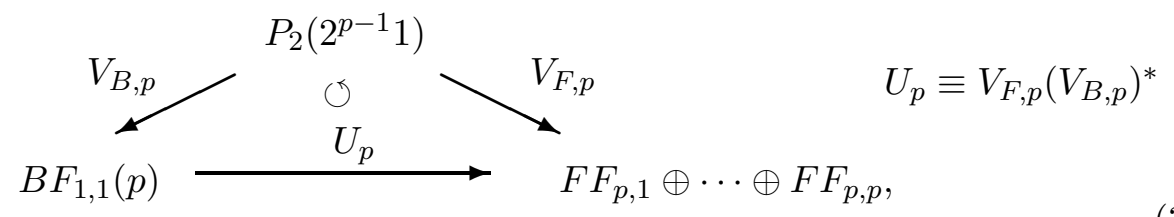

where $V_{B, p}$ denotes the unitary from $\left.P_{2}\left(2^{p-1} 1\right)\right|_{\mathcal{B}}$ to $B F_{1,1}(p)$ and $V_{F, p}$ denote the unitary from $\left.P_{2}\left(2^{p-1} 1\right)\right|_{\mathcal{A}}$ to $F F_{p, 1} \oplus \cdots \oplus F F_{p, p}$ with respect to their unitary equivalences.

Example 3.7 (i) For Example 2.7(i), if $p=1$, then we obtain the unitary $U=U_{1}$ from the Bose-Fock space to the Fermi-Fock space.

(ii) From Example 2.7(ii), $U_{2}$ is the following unitary:

$$
U_{2}: B F_{1,1}(2) \rightarrow I W \oplus I W^{*}
$$

where $I W$ and $I W^{*}$ denote the infinite wedge representation and the dual infinite wedge representation of $\mathcal{A}$, respectively.

Let $t_{1}, t_{2}$ denote canonical generators of $\mathcal{O}_{2}$ and let $\Omega$ be the GP vector of $P_{2}(1)$. For $1 \leq n_{1}<\cdots<n_{m}$ and $k_{1}, \ldots, k_{m} \in \mathbf{N}$, the RBS $\left\{b_{n}: n \in \mathbf{N}\right\}$ on $P_{2}(1)$ satisfies the following Example 3.2 in [19]:

$$
\left(b_{n_{1}}^{*}\right)^{k_{1}} \cdots\left(b_{n_{m}}^{*}\right)^{k_{m}} \Omega=\prod_{i=1}^{m} \sqrt{k_{i} !} t_{1}^{n_{1}-1} t_{2}^{k_{1}} t_{1}^{n_{2}-n_{1}} t_{2}^{k_{2}} \cdots t_{1}^{n_{m}-n_{m-1}} t_{2}^{k_{m}} \Omega .
$$


For $1 \leq k_{1}<k_{2}<\cdots<k_{l}$, the restriction of $P_{2}(1)$ on the RFS $\left\{a_{n}: n \in \mathbf{N}\right\}$ satisfies the following $([1],(3.40))$ :

$$
a_{k_{1}}^{*} \cdots a_{k_{l}}^{*} \Omega=t_{1}^{k_{1}-1} t_{2} t_{1}^{k_{2}-k_{1}-1} t_{2} \cdots t_{1}^{k_{l}-k_{l-1}-1} t_{2} \Omega .
$$

From these two formulae, it is shown that any state vector in both Fock spaces are described by using the canonical generators of $\mathcal{O}_{2}$ and $\Omega$.

According to formulae in $\S 2.1$, we show the proof of Theorem 1.3.

Proof of Theorem 1.3. (i) Since $V_{B} \Omega=\Omega_{B}$ and $V_{F} \Omega=\Omega_{F}$, we see that $U \Omega_{B}=\Omega_{F}$. From (3.11),

$$
t_{1}^{n} t_{2}^{m}=A_{n, m} t_{1}^{n+m} \quad(n, m \geq 1) .
$$

From (3.23), (3.21) and (3.22), we obtain the following:

$$
\begin{aligned}
U\left(b_{n_{1}}^{*}\right)^{k_{1}} \cdots\left(b_{n_{m}}^{*}\right)^{k_{m}} \Omega_{B} \\
\quad=C V_{F} t_{1}^{n_{1}-1} t_{2}^{k_{1}} t_{1}^{n_{2}-n_{1}} t_{2}^{k_{2}} \cdots t_{1}^{n_{m}-n_{m-1}} t_{2}^{k_{m}} \Omega \\
\quad=C A_{n_{1}-1, k_{1}} V_{F} t_{1}^{n_{1}+k_{1}-1} t_{1}^{n_{2}-n_{1}} t_{2}^{k_{2}} \cdots t_{1}^{n_{m}-n_{m-1}} t_{2}^{k_{m}} \Omega \\
\quad=C A_{n_{1}-1, k_{1}} A_{n_{2}+k_{1}-1, k_{2}} V_{F} t_{1}^{n_{2}+k_{1}+k_{2}-1} t_{1}^{n_{3}-n_{2}} t_{3}^{k_{3}} \cdots t_{1}^{n_{m}-n_{m-1}} t_{2}^{k_{m}} \Omega \\
\quad=\cdots \\
\quad=C A_{n_{1}-1, k_{1}} \cdots A_{n_{m}+k_{1}+\cdots+k_{m-1}-1, k_{m}} \Omega_{F} .
\end{aligned}
$$

Hence (1.17) holds.

(ii) From (i), we see that the particle number of both sides of (1.17) is $k_{1}+\cdots+k_{m}$. Hence the statement holds.

\section{Examples}

\subsection{State vectors in Fock representations}

We show concrete examples of Theorem 1.3. Relations among $n$-particle states for $n=1,2,3$ are shown as follows. In this subsection, we write both $\Omega_{B}$ and $\Omega_{F}$ in Theorem 1.3 as the same symbol $\Omega$ and omit the unitary operator $U$.

Example $4.1 \quad$ (i) For $n \geq 1, b_{n}^{*} \Omega=a_{n}^{*} \Omega$.

(ii) For $n, m \in \mathbf{N}$, assume $1 \leq n<m$. Then

$$
b_{n}^{*} b_{m}^{*} \Omega=a_{n}^{*} a_{m+1}^{*} \Omega, \quad\left(b_{n}^{*}\right)^{2} \Omega=\sqrt{2} a_{n}^{*} a_{n+1}^{*} \Omega .
$$


(iii) For $n, m, l \in \mathbf{N}$, assume $1 \leq n<m<l$. Then

$$
\begin{aligned}
b_{n}^{*} b_{m}^{*} b_{l}^{*} \Omega & =a_{n}^{*} a_{m+1}^{*} a_{l+2}^{*} \Omega, \\
b_{n}^{*}\left(b_{m}^{*}\right)^{2} \Omega & =\sqrt{2} a_{n}^{*} a_{m+1}^{*} a_{m+2}^{*} \Omega, \\
\left(b_{n}^{*}\right)^{2} b_{m}^{*} \Omega & =\sqrt{2} a_{n}^{*} a_{n+1}^{*} a_{m+2}^{*} \Omega, \\
\left(b_{n}^{*}\right)^{3} \Omega & =\sqrt{6} a_{n}^{*} a_{n+1}^{*} a_{n+2}^{*} \Omega .
\end{aligned}
$$

From Example 4.1, we see that state vectors of boson and fermion are similar for few particle number case.

Next, we show more general relations.

Example 4.2 (i) For each $n \geq 1$ and $m \geq 0$,

$$
\left(b_{n}^{*}\right)^{m+1} \Omega=\{(m+1) !\}^{1 / 2} a_{n}^{*} a_{n+1}^{*} \cdots a_{n+m}^{*} \Omega .
$$

(ii) For $1 \leq n_{1}<n_{2}<\cdots<n_{l}$,

$$
b_{n_{1}}^{*} \cdots b_{n_{l}}^{*} \Omega=a_{n_{1}}^{*} a_{n_{2}+1}^{*} \cdots a_{n_{l}+l-1}^{*} \Omega .
$$

In particular,

$$
b_{n}^{*} b_{n+1}^{*} \cdots b_{n+l-1}^{*} \Omega=a_{n}^{*} a_{n+2}^{*} \cdots a_{n+2(l-1)}^{*} \Omega \quad(n, l \geq 1) .
$$

From Example 4.2(i) and Theorem 1.3, the multiplicity of a mode of boson is nearly associated with the length of a block of fermions in (1.17).

We illustrate state vectors of representations of $\mathcal{O}_{2}, \mathcal{O}_{\infty}, \mathcal{A}$ and $\mathcal{B}$ in Theorem 1.3 as follows:

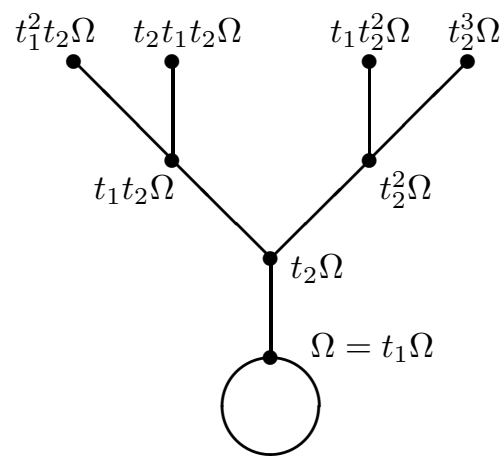

$P_{2}(1)$

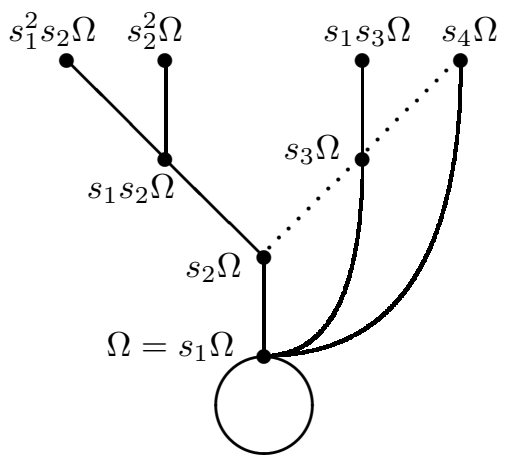

$P_{\infty}(1)=\left.P_{2}(1)\right|_{\mathcal{O}_{\infty}}$ 


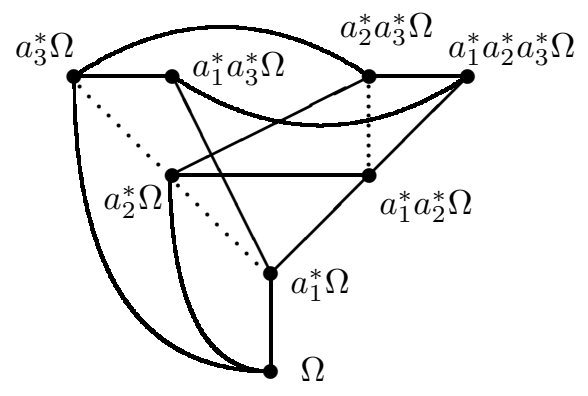

$\left.P_{2}(1)\right|_{\mathcal{A}}=$ Fermi-Fock

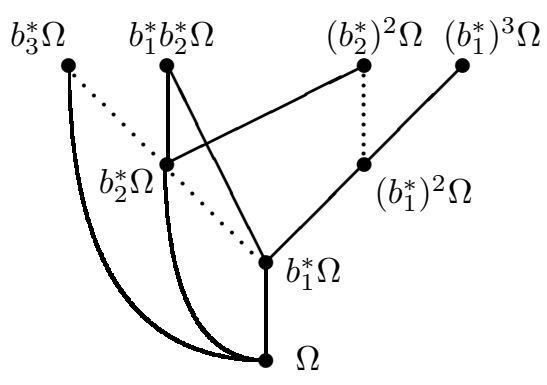

$\left.P_{2}(1)\right|_{\mathcal{B}}=\left.P_{\infty}(1)\right|_{\mathcal{B}}=$ Bose-Fock

where $P_{2}(1)$ and $P_{\infty}(1)$ are as in $\S 2$, vertices mean orthogonal vectors of the representation space of $\mathcal{O}_{2}$ and these vectors are orthogonal each other (see also graphs in $\S 3$ of [18]).

\subsection{Computation of $U^{*}$ on the Fock representation}

For $U$ in (1.16), we show the adjoint operator $U^{*}$ of $U$. Let $\mathcal{P}$ denote the set of all nonempty finite subsets of $\mathbf{N}$. For $S \in \mathcal{P}$, the block decomposition of $S$ is the partition $S=S_{1} \sqcup \cdots \sqcup S_{m}$ of $S$ such that there exists $x_{1}, \ldots, x_{m} \in S$, $k_{1}, \ldots, k_{m} \in \mathbf{N} \cup\{0\}$ which satisfy the following:

(i) $S_{i}=\left\{x_{i}, x_{i}+1, \ldots, x_{i}+k_{i}\right\}$ for each $i=1, \ldots, m$,

(ii) $x_{i}+k_{i}+1<x_{i+1}$ for each $i=1, \ldots, m-1$ when $m \geq 2$.

For $S=\left\{n_{1}, \ldots, n_{m}\right\} \in \mathcal{P}$, we write $a_{S}^{*} \equiv a_{n_{1}}^{*} \cdots a_{n_{m}}^{*}$ when $n_{1}<\cdots<n_{m}$. If $S=S_{1} \sqcup \cdots \sqcup S_{m}$ is the block decomposition of $S$, then $a_{S}^{*}=a_{S_{1}}^{*} \cdots a_{S_{m}}^{*}$. From Theorem 1.3, we obtain the action of $U^{*}$.

Proposition 4.3 For $S \in \mathcal{P}$, assume that $S=S_{1} \sqcup \cdots \sqcup S_{m}$ is the block decomposition of $S$ and $S_{i} \equiv\left\{n_{i}, \ldots, n_{i}+l_{i}\right\}$ for $i=1, \ldots, m$. Then the adjoint operator $U^{*}$ of $U$ in (1.16) is given as follows:

$$
\begin{aligned}
U^{*} \Omega_{F} & =\Omega_{B} \\
U^{*} a_{S}^{*} \Omega_{F} & =D \cdot\left(b_{n_{1}}^{*}\right)^{l_{1}+1}\left(b_{n_{2}-l_{1}-1}^{*}\right)^{l_{2}+1} \cdots\left(b_{n_{m}-\sum_{i=1}^{*-1}\left(l_{i}-1\right)}^{*}\right)^{l_{m}+1} \Omega_{B}
\end{aligned}
$$

where $D$ denotes the normalization constant given by

$$
D=\left\{\left(l_{1}+1\right) ! \cdots\left(l_{m}+1\right) !\right\}^{-1 / 2} .
$$




\subsection{Realizations of Fock spaces as $l_{2}(\mathbf{N})$}

We realize four irreducible representations $P_{2}(1), P_{\infty}(1)$ and Fock representations of bosons and fermions on the Hilbert space $l_{2}(\mathbf{N})$. Let $\left\{e_{n}: n \in \mathbf{N}\right\}$ denote the standard basis of $l_{2}(\mathbf{N})$. Then

$$
\pi\left(t_{i}\right) e_{n} \equiv e_{2(n-1)+i} \quad(i=1,2, n \in \mathbf{N})
$$

defines a representation of $\mathcal{O}_{2}$ which is $P_{2}(1)$. From Proposition $3.1(\mathrm{i}),\left.\pi\right|_{\mathcal{O}_{\infty}}$ is $P_{\infty}(1)$. This is given as follows:

$$
\pi\left(s_{m}\right) e_{n}=e_{2^{m-1}(2 n-1)} \quad(m, n \in \mathbf{N}) .
$$

We see that $\left.\pi\right|_{\mathcal{A}}$ is the Fermi-Fock space with the vacuum $e_{1}$ (see also (3.40) of [1]):

$$
\pi\left(a_{m}^{*}\right) e_{1}=e_{2^{m-1}+1}, \quad \pi\left(a_{m}\right) e_{1}=0 \quad(m \geq 1) .
$$

Furthermore, we see that $\left.\pi\right|_{\mathcal{B}}$ is the Bose-Fock space with the vacuum $e_{1}$ :

$$
\pi\left(b_{m}^{*}\right) e_{1}=e_{2^{m-1}+1}, \quad \pi\left(b_{m}\right) e_{1}=0 \quad(m \geq 1) .
$$

\section{References}

[1] Abe, M. and Kawamura, K., "Recursive fermion system in Cuntz algebra. I - Embeddings of fermion algebra into Cuntz algebra-," Commun. Math. Phys. 228, 85-101 (2002).

[2] Abe, M. and Kawamura, K., "Nonlinear transformation group of CAR fermion algebra," Lett. Math. Phys. 60, 101-107 (2002).

[3] Abe, M. and Kawamura, K., "Recursive fermion system in Cuntz algebra. II - Endomorphism, automorphism and branching of representation -," Report No. RIMS-1362, 2002 (unpublished).

[4] Abe, M. and Kawamura, K., "Pseudo Cuntz algebra and recursive FP ghost system in string theory," Int. J. Mod. Phys. A 18, 607-625 (2003).

[5] Abe, M. and Kawamura, K., "Branching laws for endomorphisms of fermions and the Cuntz algebra $\mathcal{O}_{2}$," J. Math. Phys. 49, 043501-01043501-10 (2008).

[6] Ambjorn, J., Karakhanyan, D., Mirumyanb, M. and Sedrakyana, A., "Fermionization of the spin- $S$ Uimin-Lai-Sutherland model: generalisation of the supersymmetric $t-J$ model to spin- $S$," Nuclear Phys. B 599, 547-560 (2001). 
[7] Bratteli, O. and Jorgensen, P. E. T., "Iterated function systems and permutation representations of the Cuntz algebra," Memoirs Amer. Math. Soc. 139, 1-89 (1999).

[8] Bratteli, O. and Robinson, D. W., Operator algebras and quantum statistical mechanics 2 (Springer, New York, 1981).

[9] Coleman, S., "Quantum sine-Gordon equation as the massive Thirring model," Phys. Rev. D 11, 2088-2097 (1975).

[10] Cuntz, J., "Simple $C^{*}$-algebras generated by isometries," Commun. Math. Phys. 57, 173-185 (1977).

[11] Davidson, K. R. and Pitts, D. R., "The algebraic structure of noncommutative analytic Toeplitz algebras," Math. Ann. 311, 275-303 (1998).

[12] Davidson, K. R. and Pitts, D. R., "Invariant subspaces and hyperreflexivity for free semigroup algebras," Proc. London Math. Soc. 78, 401-430 (1999).

[13] Gopakumar, R. and Gross, D. J., "Mastering the master field," Nuclear Phys. B 451, 379-415 (1995).

[14] Ilieva, N. and Thirring, W., "Anyons and the Bose-Fermi duality in the finite-temperature Thirring model," Theor. Math. Phys. 121(1), 1294-1314 (1999).

[15] Kac, V. G., Infinite dimensional Lie algebras, 3rd ed. (Cambridge University Press, 1990).

[16] Kashiwara, M. and Schapira, P., Categories and sheaves (SpringerVerlag, Berlin Heidelberg New York, 2005).

[17] Kawamura, K., "Generalized permutative representations of the Cuntz algebras," e-print arXiv:math.OA/0505101.

[18] Kawamura, K., "Extensions of representations of the CAR algebra to the Cuntz algebra $\mathcal{O}_{2}$ - the Fock and the infinite wedge -, J. Math. Phys. 46, 073509-1-073509-12 (2005).

[19] Kawamura, K., "Recursive boson system in the Cuntz algebra $\mathcal{O}_{\infty}$," J. Math. Phys. 48, 093510-1-093510-16 (2007). 
[20] Kopietz, P., Bozonization of interacting fermions in arbitrary dimensions (Springer-Verlag, Berlin Heidelberg, 1997).

[21] Mandelstam, S., "Soliton operators for the quantized sine-Gordon equation," Phys. Rev. D 11, 3026-3030 (1975).

[22] Miwa, T., Jimbo, M. and Date, E., Solitons: Differential equations, symmetries and infinite dimensional algebras (Cambridge University Press, Cambridge, 2000).

[23] Nakanishi, N. and Ojima, I., Covariant operator formalism of gauge theories and quantum gravity (World Scientific, Singapore, 1990).

[24] Okounkov, A., "Infinite wedge and random partitions," Selecta Math. New Ser. 7, 57-81 (2001).

[25] Peskin, M. E. and Schroeder, D. V., An introduction to quantum field theory (Addison-Wesley, Publishing Company,1995).

[26] Sakurai, J. J.; Tuan, S. F. editor, Modern quantum mechanics. Revised edit. (Addison-Wesley, Publishing Company, 1994).

[27] Stone, M., Bosonization (World Scientific, Singapore, 1994). 